\title{
One of the earliest studies publishing sequencing data (Illumina) from two SARS-Cov2 patients in Wuhan failed to identify potentially pathogenic bacteria using the program Metaphlan2, when they clearly exist
}

Sandeep Chakraborty

\section{Letter}

It is increasingly being evident that bacterial coinfection is playing an important role in SARS-Cov2 [1-4], explaining hydroxychloroquine and azithromycin working in clinical trials [5]. Previously, I have analyzed the metagenomes in patients from China [6,7], San Diego [8] and Brazil [9] - showing many bacterial species (sometimes many unknowns), and Prevotella immune-supression genes taking over [6].

\section{Failure to identify pathogenic bacteria using Metaphlan2}

One of the earliest studies publishing sequencing data (Illumina) from two SARS-Cov patients in Wuhan [10] wrote 'bacterial pathogen identification was carried out by using the Metaphlan2 program, which revealed Capnocytophaga sp and Veillonella sp in sample 2 and none in sample 1, and both bacteria identified were not known for their pathogenicity. Collectively, coronavirus is likely to be the main microbial pathogen' [10]. This is clearly wrong - Capnocytophaga and Lautropia are there, and also so many other pathogenic species (Table 1). Sequences and full list of bacterial species are in SIstudy3MNGS. And Capnocytophaga, found in the saliva of humans, dogs and cats can be pathogenic [11]. Another observation is that bacteria will high counts are mostly anaerobic.

Table 1: Bacterial species identified in two patients from Wuhan: The original paper stated that 'bacterial pathogen identification was carried out by using the Metaphlan2 program, which revealed Capnocytophaga sp and Veillonella sp in sample 2 and none in sample 1, and both bacteria identified were not known for their pathogenicity' This is clearly wrong - Capnocytophaga and Lautropia are there, but there are so many other pathogenic species. Also Capnocytophaga, found in the saliva of humans, dogs and cats can be pathogenic [11]. There are many more species, just listed top 10 here.

\begin{tabular}{|c|c|c|}
\hline & Patient1 & Patient2 \\
\hline Accid: Total & SRR10903402 676694 & SRR10903401 476632 \\
\hline \hline & Bacteria/ Reads per million & Bacteria/ Reads per million \\
\hline 1 & Capnocytophaga 1195.6 & Prevotella 247 \\
2 & Streptococcus 694.4 & Lautropia 236 \\
3 & Prevotella 578.9 & Veillonella 132 \\
4 & Lautropia 512.4 & Escherichia 17.5 \\
5 & Lactobacillus 161.7 & Streptococcus 16.5 \\
6 & Veillonella 157.5 & Campylobacter 14 \\
7 & Mycoplasma 107.1 & Megasphaera 4.5 \\
8 & Schaalia 81.9 & Acinetobacter 3.5 \\
9 & Escherichia 49 & Actinomyces 3.5 \\
\hline
\end{tabular}




\section{References}

1. Zhu N, Zhang D, Wang W, Li X, Yang B, et al. (2020) A novel coronavirus from patients with pneumonia in China, 2019. New England Journal of Medicine .

2. Chan JFW, Yuan S, Kok KH, To KKW, Chu H, et al. (2020) A familial cluster of pneumonia associated with the 2019 novel coronavirus indicating person-to-person transmission: a study of a family cluster. The Lancet .

3. Perlman S (2020). Another decade, another coronavirus.

4. Wu F, Zhao S, Yu B, Chen Y, Wang W, et al. (2020) Complete genome characterisation of a novel coronavirus associated with severe human respiratory disease in Wuhan. China bioRxiv 24.

5. Gautret P, Lagier JC, Parola P, Meddeb L, Mailhe M, et al. (2020) Hydroxychloroquine and azithromycin as a treatment of covid-19: results of an open-label non-randomized clinical trial. International Journal of Antimicrobial Agents : 105949.

6. Chakraborty S (2020). The 2019 Wuhan outbreak could be caused by the bacteria Prevotella, which is aided by the coronavirus, possibly to adhere to epithelial cells - prevotella is present in huge aemounts in patients from both China and Hong Kong. doi:10.31219/osf.io/usztn. URL osf .io/usztn.

7. Chakraborty S (2020). Metagenome of sars-cov2 patients in shenzhen with travel to wuhan shows a wide range of species - lautropia, cutibacterium, haemophilus being most abundant - and campylobacter explaining diarrhea. doi:10.31219/osf.io/jegwq. URL osf .io/jegwq.

8. Chakraborty S (2020). San Diego county Nanopore SARS-Cov2 sequencing data shows metagenomic Prevotella, Haemophilus parainfluenzae, a lot of unknown species and chimeric reads. doi: 10.31219/osf.io/cvbqf. URL osf .io/cvbqf.

9. Chakraborty S (2020). Metagenome of sars-cov2 from a patient in brazil shows a wide range of bacterial species - lautropia, prevotella, haemophilus - overshadowing viral reads, which does not even add up to a full genome, explaining false negatives. doi:10.31219/osf.io/2xt3w. URL osf .io/2xt3w.

10. Chen L, Liu W, Zhang Q, Xu K, Ye G, et al. (2020) RNA based mNGS approach identifies a novel human coronavirus from two individual pneumonia cases in 2019 Wuhan outbreak. Emerging Microbes \& Infections 9: 313-319.

11. Hundertmark M, Williams T, Vogel A, Moritz M, Bramlage P, et al. (2019) Capnocytophaga canimorsus as cause of fatal sepsis. Case reports in infectious diseases 2019. 